\title{
Positive classical gravitational energy from classical supergravity
}

\author{
S. Deser \\ Physics Department, Brandeis University, Waltham, Massachusetts 02254
}

(Received 28 February 1983)

\begin{abstract}
Classical gravity has sufficient supersymmetric extension that its Hamiltonian is expressible as a Poisson bracket of supercharges. Its value, the energy, is then a manifestly positive functional of the spinor transformation parameter, identical in form to Witten's expression whose supergravity basis is thereby established.
\end{abstract}

\section{INTRODUCTION}

After several decades of attempts (for a history, see Ref. 1), positivity of classical gravitational energy was finally demonstrated recently, and in three different ways. The first was a proof of positivity (for arbitrary $N$ ) of (quantum) supergravity energy, ${ }^{2}$ followed by a classical-limit argument ${ }^{3}$ to general relativity. Since the classical limit keeps only tree diagrams and the formal proof is valid for arbitrary $\hbar$, the classical result is valid irrespective of "existence" questions about the quantum theory. The second was a mathematically rigorous argument ${ }^{4}$ based on differential geometry. The third ${ }^{5}$ exhibited an extremely simple, elegant, and manifestly positive expression for energy, and is also rigorous. ${ }^{6}$ Our purpose is not to provide yet another proof, but rather to connect the first and third ideas. This is of some importance, because although Witten's proof ${ }^{5}$ was motivated by supergravity, but attempting to avoid the two steps of the first result, it does not appear to have any connection either with supergravity or other aspects of classical relativity. We shall show that there exists an extremely direct derivation of Witten's expressions from "classical" supergravity (defined below); the steps we shall take actually correspond closely to those used in Ref. 2, with a simple change of name from commutation to Poisson bracket (PB) relations. A derivation of this connection was recently independently obtained by Horowitz and Strominger, ${ }^{7}$ with some differences in interpretation.

The general framework is the following. First, we show that source-free classical relativity is a locally supersymmetric point of the Einstein-RaritaSchwinger system in which all fields are classical, obeying PB (or anti-PB) relations; the spin- $\frac{3}{2}$ fields $\psi_{\mu}$ are Grassmann rather than Clifford elements. Our final results will be for the gravitational field alone, and we shall set $\psi_{\mu}=0$ at the end; effectively, we will be obtaining information about "real" functions by a detour through the "complex" domain. From the kinematical fact that the PB of two supersymmetry generators $Q$ on the metric is a translation, we will express the time translation generator, (i.e., the energy) as a square of supersymmetry transformations. We shall only need the form of these generators as they act on the fermionic variables. All our operations involve only an initialvalue surface since both energy and supercharge are entirely defined there. In this equal-time framework, the form of $Q$ becomes manifest once the (linear) constraint on the fields is solved, since we know how the $\psi_{\mu}$ transform and the fundamental PB among the dynamical variables. The last step is simply to recognize the Witten equations in the PB of the supercharges, thereby also identifying his spinor with the local supersymmetry parameter. We emphasize that the difficult point of rigor in the Witten proof, analysis of the solutions of his basic equation, is not avoided by our method, but fortunately all that has been done for us in the original papers. 5,6

\section{CLASSICAL SUPERGRAVITY}

Adjoin to the classical Einstein action the Rarita-Schwinger action, whose field $\psi_{\mu}$ is classical but Grassmann valued, ${ }^{8}$ still obeying $R^{\mu} \equiv \epsilon^{\mu \nu \alpha \beta} \gamma_{5} \gamma_{\nu} D_{\alpha} \psi_{\beta}=0$. This coupled system is invariant under the usual local transformations

$$
\begin{aligned}
& \delta e_{\mu a}=i \bar{\alpha} \gamma_{a} \psi_{\mu}, \quad \delta \psi_{\mu}=2 D_{\mu} \alpha, \\
& \delta g_{\mu \nu}=i \bar{\alpha}\left(\gamma_{\mu} \psi_{v}+\gamma_{\nu} \psi_{\mu}\right) .
\end{aligned}
$$

Furthermore, at $\psi_{\mu}=0$, iteration of the above transformation on $g_{\mu \nu}$ itself is a pure local translation,

$$
\left[\delta_{1}, \delta_{2}\right] g_{\mu v}=\left(2 i \bar{\alpha}_{1} \gamma^{\alpha} \alpha_{2}\right) \partial_{\alpha} g_{\mu v}
$$


where the local time translation parameter $\delta t$ in particular is proportional to $\left(\alpha_{1} \alpha_{2}\right)$. (Note that although we are dealing only with infinitesimal $\alpha$ parameters, this will suffice to deduce the properties of the full energy.) We must next obtain the form of the generators of (1) in terms of the system's dynamical variables; however, because we only want the $\psi_{\mu}=0$ part after performing (2), we only need to concentrate on their $\psi$ dependence (their effect on $g_{\mu \nu}$ will be automatically correct if they are expressed covariantly). Also, we will be working on an (arbitrary) initial-value surface $t=0$ throughout. That is, we will be concerned only with the initialdata set and only to first order in $\psi$ :

$$
G_{0 \mu}=0, \quad R^{0}=\sigma^{i j 4} D_{i} \psi_{j}=0,
$$

where ${ }^{4} D_{i}$ are the spatial components of the covariant derivative $D_{\mu}$. For compactness, and to be able to exhibit all steps, we consider the special (but fully nonlinear) case in which the gravitational field momenta ${ }^{9} \pi^{i j}$ vanish. Then the $G_{0 \mu}=0$ constraints reduce to one equation $\left(G_{00}=0\right)$ which states that the intrinsic spatial curvature of the initial surface is trace-free, and the covariant derivative ${ }^{4} D_{i}$ in (3) reduces to ${ }^{3} D_{i}$, the one with respect to the only gravitational components remaining, the spatial metric $g_{i j}$ :

$$
{ }^{3} R=0, \quad \sigma^{i j 3} D_{i} \psi_{j}=0 .
$$

The $\psi_{i}$ field is thus effectively a free field in a prescribed curved space. We now need to analyze the constraint $R^{0}$ in order to find the dynamical part of the spatial components $\psi_{i}$ in the ${ }^{3} R=0$ background, with the usual boundary conditions required for energy to be defined, $g_{i j} \sim \delta_{i j}+O(1 / r)$ and $\psi_{i} \sim O\left(1 / r^{2}\right)$ at spatial infinity. Let us first recall what happens in flat space ${ }^{10}$ : Decomposing the three spinors $\psi_{i}$ into their orthogonal irreducible parts according to

$$
\begin{aligned}
& \psi_{i}=\psi_{i}^{T t}+\kappa_{i} \eta+\partial_{i} \beta, \quad 2 \kappa_{i}=\gamma^{j}\left(\delta_{i j} \nabla^{2}-\partial^{2}{ }_{i j}\right), \\
& \kappa \cdot \partial=\partial \cdot \kappa=0, \quad \gamma \cdot \psi^{T t}=0=\partial \cdot \psi^{T t},
\end{aligned}
$$

the constraint $\sigma^{i j} \partial_{i} \psi_{j}=0$ involves only the variable $\eta$ and states that $\partial \eta=0$; the boundary conditions then imply that $\eta$ vanishes. The gauge variable $\beta$ is irrelevant, as it has no dynamics (or PB relations). This leaves as dynamical variable $\psi^{T t}$ (and its conjugate momentum $\pi^{T t}$ ). Although this component is gauge invariant by definition, note that we have not fixed a gauge (e.g., $\gamma \cdot \psi=0$ ) but simply found that the action is independent of $\beta$ (just as in electrodynamics, whose action only depends on $A_{i}^{T}$ ). However, one may still discuss gauge $\left(\delta \psi_{i}=2 \partial_{i} \epsilon\right)$ transformations on $\psi_{i}$ in the reduced space of the dynamical $\psi_{i}^{T t}$. In view of the canonical (anti-) PB relations $^{11}$

$$
\left\{\pi^{T t i}(\overrightarrow{\mathrm{r}}), \psi_{j}^{T t}(0)\right\}=\left[\delta_{j}^{i}(\overrightarrow{\mathrm{r}})\right]^{T t},
$$

the generator is clearly given by

$$
\begin{aligned}
Q & =\int d^{3} x\left(\pi^{T t i} 2 \partial_{i} \epsilon+i \partial^{i} \epsilon \psi_{i}^{T t}\right) \\
& =\oint d S_{i}\left(\pi^{T t i} 2 \epsilon+i \epsilon \psi_{i}^{T t}\right)
\end{aligned}
$$

where the second form is obtained by partial integration. Because of their $T t$ properties, the dynamical variables in (7) only retain those parameters $\epsilon$ which obey the Dirac and Laplace equations, i.e., such that $\gamma^{i}\left(\partial_{i} \epsilon\right)=0=\partial^{i}\left(\partial_{i} \epsilon\right)$. But the only harmonic function in flat space is a constant $\epsilon_{0}$ so the $\psi_{i}$ field in flat space remains untransformed. Things change, however, when we go to a curved background. Here $\psi_{i}$ is still a free field, and we may still decompose it as follows:

$$
\begin{aligned}
& \psi=\psi_{i}^{T t}+\kappa_{i} \eta+D_{i} \beta, \\
& 2 \kappa_{i} \equiv \gamma_{i} D^{2}-\frac{1}{2}\left(D_{i} \not D+\not D D_{i}\right)-\frac{1}{4} R_{i j} \gamma^{j}, \\
& \kappa \cdot D=D \cdot \kappa=0, \quad \gamma \cdot \psi^{T t}=0=D \cdot \psi^{T t} .
\end{aligned}
$$

It can be shown, using the Ricci identities on any spinor (see the Appendix) that the full orthogonality of (5) still holds, and that the covariant constraint $\sigma^{i j} D_{i} \psi_{j}=0$ still annuls $\eta$, while gauge invariance ensures that $\beta$ never enters in the dynamics. It is now obvious that the generator of $\delta \psi_{i}=2 D_{i} \epsilon$ is given by

$$
Q_{V}=\int d^{3} x\left(\pi^{T t i} 2 D_{i} \epsilon+i D^{i} \epsilon \psi_{i}^{T t}\right)
$$

or

$$
Q_{S}=\oint d S_{i}\left(\pi^{T t i} 2 \epsilon+i \epsilon \psi^{T t i}\right),
$$

where the covariant $T t$ property has been used to write the surface form. This time, however, $\epsilon$ must satisfy the covariant equations

$$
\not D \epsilon \equiv \gamma^{i}\left(D_{i} \epsilon\right)=0, \quad \nabla^{2} \epsilon \equiv D^{i}\left(D_{i} \epsilon\right)=0 \text {. }
$$

Because $\not D \epsilon=\nabla^{2} \epsilon$ when ${ }^{3} R=0$, all we need is the Dirac equation whose solutions are still asymptotically nonzero (there are no asymptotically vanishing harmonic functions in an elliptic space either). But since the metric is nontrivial, the solutions now read

$$
\epsilon=\epsilon_{0}\left[1+f(\theta, \phi) / r+O\left(1 / r^{2}\right)\right],
$$

and $D_{i} \epsilon \neq 0$; there are no Killing spinors in a general curved space. The $Q$ generators are therefore nontrivial, and we immediately find that

$$
\begin{aligned}
& {\left[Q_{V}, Q_{V}\right]_{\mathrm{PB}}=4 i \int d^{3} x|D \epsilon|^{2},} \\
& {\left[Q_{V}, Q_{S}\right]_{\mathrm{PB}}=4 i \oint d S_{i} \epsilon D^{i} \epsilon .}
\end{aligned}
$$


But, as shown in Ref. 5, the right side of (12) is just $2 i\left|\epsilon_{0}\right|^{2} E$ where $E$ is the energy of the gravitational field. This completes our derivation, since the set (10), (11), (12) is precisely that of Witten. The quantity $2 i\left|\epsilon_{0}\right|^{2}$ is the constant infinitesimal time translation (times two constant Grassmann elements which multiply everything). In the generic case $G_{0 \mu}=0$, (11), and (12) are unchanged, except that $D_{i}$ is ${ }^{4} D_{i}$, while the value of (12) becomes $2 i\left(\epsilon_{0} \gamma^{0} \gamma_{\mu} \epsilon_{0}\right) P^{\mu}$. We emphasize that there is no interior gauge fixing involved here, but that the asymptotic boundary conditions which are essential to define energy ${ }^{12}$ lead to a unique interior supersymmetry transformation, parametrized by $\epsilon_{0}$. The gravitational Hamiltonian generates the (constant) global time translation $\delta t=2 i\left|\epsilon_{0}\right|^{2}$ on the metric at every interior point, just as a Hamiltonian should.

We have used the explicit reduction to canonical, unconstrained variables here because the $\psi$ system is sufficiently Abelian. One could have followed the alternate Dirac bracket method of dealing with the gauges and constraints, as was done in Ref. 2 at the quantum level and in Ref. 7 classically. Indeed, if in Ref. 2 a factor $\epsilon$ is included in the supercharge of Eq. (2), and the PB version of the basic anticommutator Eq. (7) there is taken, one recovers the Witten system; the $D \epsilon=0$ equation is there seen as a consequence of the $\gamma \cdot \psi=0$ gauge fixing. In the canonical method, no gauge choice need be made; the $D \epsilon=0=D^{2} \epsilon$ conditions are simply the (mutually compatible) restrictions on gauge changes within the physical $(T t)$ subspace, and reflect the "zerofrequency" ambiguity in the $T t$ decomposition $\left(D_{i} \epsilon\right.$ is a $T t$ vector if $D \epsilon=0$ ) when there is a nontrivial background geometry. By contrast, we saw that in flat space $\partial \epsilon=0$ implies $\epsilon=\epsilon_{0}$ and so $\delta \psi_{i}=2 \partial_{i} \epsilon_{0}=0$.

The above results may be generalized to include matter coupling, by using the same supersymmetrizing technique for the bosonic systems, then setting their fermionic companions to zero at the end. We recall that all supersymmetrizable boson actions have positive energy density $T_{M}^{00}$, while classical fermions never do, so positivity will hold exactly as far as it should. The constraints (4) acquire $T_{M}^{00}$ and $j^{0}$ sources, where $j^{\mu}$ is the matter supercurrent. By virtue of the matter field $\mathrm{PB}$, it follows that $\left\{j^{0}(r), j^{0}(0)\right\}=2 i T_{M}^{00} \delta^{3}(r)$, and that the matter supercharge $Q_{M}=\int d^{3} x \bar{\epsilon} j^{0}$ generates local supersymmetry transformations on the matter variables. Since the theory is no longer manifestly gauge independent in the presence of dynamical sources, we specify the $\gamma \cdot \psi=0$ gauge to make the $\epsilon$ parameter obey Witten's $\emptyset \epsilon=0$ condition. This gauge choice is only an apparent restriction, since the value of the energy (or supercharge), unlike its form in terms of dynamical variables, is gauge invariant: Positivity need only be established in one gauge. It is most convenient to use the Dirac bracket ${ }^{2,7}$ formalism rather than explicitly solving the constraint here, so that everything is formally expressed in terms of the full $\psi_{i}$, which is implicitly subject to the gauge and constraint conditions. The total supercharge must still be expressible as a flux integral when sources are present, and we shall see that the obvious counterpart,

$$
Q_{S}=\oint d S_{i}\left(\pi^{i} 2 \epsilon+i \epsilon \psi^{i}\right)
$$

of $(9 b)$ is correct.

In our gauge, the constraint becomes $\sigma^{i j} D_{i} \psi_{j}=-\frac{1}{2} D \cdot \psi=-(i / 4) \gamma_{0} j^{0}$. The corresponding volume expression for $Q$ is

$$
\begin{aligned}
Q_{V} & =\int d^{3} x D_{i}\left(\pi^{i} 2 \epsilon+i D^{i} \epsilon \psi^{i}\right) \\
& =\int d^{3} x\left(\pi^{i} 2 D_{i} \epsilon+i D^{i} \epsilon \psi_{i}+\bar{\epsilon} j^{0}\right)
\end{aligned}
$$

The last form is obtained by using the constraint. In this formalism the $(\pi, \psi)$ have effective unit $\mathrm{PB}$, so it is manifest that $Q_{V}$ correctly generates the proper supersymmetry rotations on both $\psi_{i}$ and matter fermions. The generalization of (11) and (12) now follows directly from (13) and the various PB relations:

$$
\begin{aligned}
& {\left[Q_{V}, Q_{V}\right]=4 i \int d^{3} x\left(|D \epsilon|^{2}+\frac{1}{2} T_{M}^{0}|\epsilon|^{2}\right),} \\
& {\left[Q_{V}, Q_{S}\right]=4 i \oint d S_{i} \epsilon D^{i} \epsilon .}
\end{aligned}
$$

The right-hand side of (15) is identical to that of (12), because the total energy is still read off from the asymptotics of the metric. The set (14) and (15) is the Witten energy form in the presence of matter. We emphasize that there is no real loss of generality in the fact that, while always correct, (14) has only been derived here for supersymmetrizable systems, since most classically interesting cases such as vector fields, scalars, or point particles are included.

Finally, we add that the same technique also provides a direct proof of positive energy in the presence of a negative (anti-de Sitter) cosmological constant $-\Lambda$ (Refs. 12 and 13). All the above steps are repeated, with the background constraint ${ }^{3} R+2 \Lambda=0$ and all $D_{i}$ replaced by the standard $\widetilde{D}_{i}=D_{i}+\frac{1}{2} \sqrt{\Lambda / 3} \gamma_{i}$ which have the property $\sigma^{i j} \widetilde{D}_{i} \widetilde{D}_{j} \lambda=0$. We have not checked the difficult part, the solutions of $\widetilde{D} \epsilon=0$, but they must work out in exactly the same way as when $\Lambda=0$. There are undoubtedly further applications of these methods to explain other properties of classical gravity. They should also permit a demonstration that higherderivative theories (of the $R+R^{2}$ type) do not have 
positive energy because they contain ghost degrees of freedom whose associated fermionic modes have fundamental anti-PB relations of opposite sign and hence yield $\left(-Q^{2}\right)$ contributions. ${ }^{14}$

\section{ACKNOWLEDGMENTS}

I thank the Institute of Theoretical Physics, Santa Barbara, for hospitality while this work was completed. This research was supported in part by NSF Grant No. PHY 82-01094.

\section{APPENDIX}

We give here some details of the properties of the covariant orthogonal decomposition (8) which underlies our derivation. As a preface, note that the naive breakup $\psi_{i}=\psi_{i}^{T t}+\gamma_{i} \lambda+D_{i} \beta$ would really suffice for our purposes, even though $\gamma^{i}$ and $D_{i}$ are not orthogonal. As before, the $D_{i} \beta$ part never enters the constraint or the action; it is only needed to "balance" the three $\psi_{i}$ components with the three spinors $\psi_{i}^{T t}, \lambda$, and $\beta$ (remembering that $\psi_{i}^{T t}$ has only one component). The constraint requires $D \lambda=0$, which implies $\lambda=0$ with our $1 / r^{2}$ boundary conditions on $\psi_{i}$. (The $D_{i} \beta$ part obeys $D \beta=\gamma \cdot \psi$ and
$D^{2} \beta=D \cdot \psi$, which are compatible for $\lambda=0$.) The form (8) has the more elegant orthogonality properties $\kappa \cdot D=D \cdot \kappa=\kappa \cdot \psi^{T t}=D \cdot \psi^{T t}=0$. The constraint implies that $\not D D^{2} \eta$ vanishes, which means $\not \eta=0$ with our boundary conditions. This can be verified to imply that $\kappa_{i} \eta$ itself vanishes. All the above properties depend on the Ricci identities and on the fact that $D^{i} R_{i j}=0=R_{i j} \gamma^{i} \gamma^{j}$ because ${ }^{3} R=0$. Our conventions are as follows:

$$
\begin{array}{ll}
R_{i j k}{ }^{l} \sim+\Gamma_{j k, i}^{l}, & R_{i j}=R_{i k j}{ }^{k}, \\
\sigma^{i j}=\frac{1}{4}\left[\gamma^{i}, \gamma^{j}\right], & {\left[\gamma^{i}, \sigma^{j k}\right]=\delta^{j i} \gamma^{k}-\delta^{k i} \gamma^{j} .}
\end{array}
$$

The Ricci identities on a spinor and vector are, respectively,

$$
\begin{aligned}
& {\left[D_{i}, D_{j}\right] \lambda=-\frac{1}{2} R_{i j a b} \sigma^{a b} \lambda,\left[D_{i}, D_{j}\right] V^{j}=R_{i j} V^{j},} \\
& \sigma^{i j}\left[D_{i}, D_{j}\right] \lambda=\frac{1}{4} R \lambda .
\end{aligned}
$$

In checking the various orthogonality properties, it is important to recall that $\left(D_{i} \eta\right)$, say, is to be differentiated as a vector-spinor, unlike $\eta$ itself. Correspondingly, the general form (8) of $\kappa^{i}$ must be used, unless it acts on a spinor, where it reduces to $2 \kappa_{i} \eta=\left(\gamma_{i} D^{2}-D_{i} \not\right) \eta$.
${ }^{1}$ D. R. Brill and P. S. Jang, in General Relativity and Gravitation, edited by A. Held (Plenum, New York, 1980); J. York, in Essays in General Relativity, edited by F. Tipler (Academic, New York, 1980).

${ }^{2}$ S. Deser and C. Teitelboim, Phys. Rev. Lett. 39, 249 (1977).

${ }^{3}$ M. T. Grisaru, Phys. Lett. $\underline{73 B}, 207$ (1978).

${ }^{4}$ R. M. Schoen and S. T. Yau, Commun. Math. Phys. 79, 231 (1981); 65, 45 (1979); Phys. Rev. Lett. 43, 1457 (1979).

${ }^{5}$ E. Witten, Commun. Math. Phys. $\underline{80}, 381$ (1981).

${ }^{6}$ T. Parker and C. H. Taubes, Commun. Math. Phys. $\underline{84}$, 223 (1982); O. Reula, J. Math. Phys. 23, 810 (1982).

${ }^{7} \mathrm{G}$. Horowitz and A. Strominger, preceding paper, Phys. Rev. D 27, 2793 (1983). See also C. M. Hull, Cam- bridge report, 1983 (unpublished).

${ }^{8}$ R. Casalbuoni, Nuovo Cimento 33A, 115 (1976); 33A, 389 (1976).

${ }^{9}$ R. Arnowitt, S. Deser, and C. W. Misner, in Gravitation, edited by L. Witten (Wiley, New York, 1962).

${ }^{10}$ S. Deser, J. H. Kay, and K. S. Stelle, Phys. Rev. D $\underline{16}$, 2448 (1977); G. Senjanovic, ibid. 16, 307 (1977).

${ }^{11}$ The canonical momentum is essentially $(i / 2) \psi_{i}^{T t}$; details on this and the $T t \delta$ function may be found in the first paper of Ref. 10.

${ }^{12}$ L. Abbott and S. Deser, Nucl. Phys. B195, 76 (1982).

${ }^{13} \mathrm{G}$. Gibbons, S. Hawking, G. Horowitz, and M. Perry, Commun. Math. Phys. (to be published).

${ }^{14}$ D. Boulware, S. Deser, G. Gibbons, and K. Stelle (unpublished). 